\title{
Soluble CD163 was linked to galectin-3, diabetic retinopathy and antidepressants in type 1 diabetes
}

\author{
Eva O Melin ${ }^{1,2,3}$, Jonatan Dereke ${ }^{4}$, Maria Thunander1,2,5 and Magnus Hillman ${ }^{4}$ \\ ${ }^{1}$ Department of Clinical Sciences, Endocrinology and Diabetes, Lund University, Lund, Sweden \\ ${ }^{2}$ Department of Research and Development, Växjö, Sweden \\ 3Primary Care, Växjö, Sweden \\ ${ }^{4}$ Department of Clinical Sciences Lund, Diabetes Research Laboratory, Lund University, Lund, Sweden \\ ${ }^{5}$ Department of Internal Medicine, Endocrinology and Diabetes, Central Hospital, Växjö, Sweden \\ Correspondence should be addressed to E O Melin: eva.melin@kronoberg.se
}

\begin{abstract}
Objective: Depression has been associated with diabetic retinopathy and increased plasma levels of galectin-3, a lectin expressed in activated macrophages. Increased levels of sCD163, the soluble form of a macrophage expressed scavenger receptor involved in several inflammatory processes, have been demonstrated in the vitreous of the eye in type 1 diabetes (T1D) patients with severe diabetic retinopathy. The aim was to explore whether circulating SCD163 was associated with diabetic retinopathy, depression and/ or galectin-3 in T1D patients, controlling for gender, metabolic factors, other diabetes complications, life style and medication.

Design: Cross sectional.

Methods: Two hundred eighty-seven T1D patients, men $56 \%$, age 18-59 years, diabetes duration $\geq 1$ year, were consecutively recruited from one specialist diabetes clinic.

Depression was assessed by Hospital Anxiety and Depression Scale-Depression subscale. Blood samples, anthropometrics and blood pressure values were collected, supplemented with data from electronic medical records and the Swedish National Diabetes Registry. High plasma SCD163 was defined as $\geq 0.575 \mathrm{mg} / \mathrm{L}$ (corresponding to the 80th percentile) and high plasma galectin-3 as $\geq 4.659 \mu \mathrm{g} / \mathrm{L}$ (corresponding to the 95 th percentile). Results: The prevalence of depression was $10 \%$, antidepressant medication $8 \%$, diabetic retinopathy $72 \%$, high sCD163 20\% and high galectin $35 \%$. High galectin-3 (AOR 9.7), antidepressants (AOR 3.8), diabetic retinopathy (AOR 2.4) and systolic blood pressure (per $\mathrm{mmHg}$ ) (AOR 1.03) were associated with high SCD163.

Conclusions: This is the first study to show that circulating SCD163 was independently associated with galectin-3, the use of antidepressants and diabetic retinopathy, in patients with T1D. Depression was not associated with sCD163.
\end{abstract}

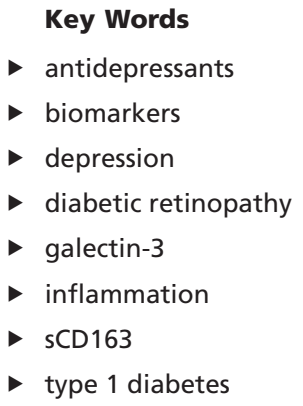

Endocrine Connections (2018) 7, 1343-1353

\section{Introduction}

Diabetic retinopathy (DR) is the most common complication of diabetes $(1,2)$ and is a major cause of blindness $(3,4)$. DR progresses over time, but fortunately only a limited number of patients acquire one of the two major vision-threatening complications, proliferative DR or macular edema (3). The necessity of optimal metabolic control in the prevention of DR is well documented, but some patients develop vision loss despite good metabolic
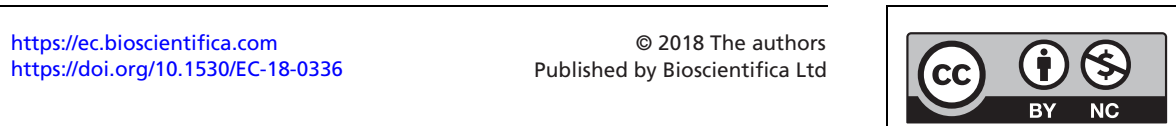
control $(2,3,5)$. The pathogenesis of DR occurs by alterations in multiple biochemical pathways, which involve several metabolic, inflammatory and endocrine responses $(1,2,4,6)$.

Depression is the most common psychiatric disorder worldwide with great impact not only on functioning and quality of life, but also on somatic health due to metabolic, inflammatory, autonomic and hypothalamicpituitary-adrenal (HPA) axis dysregulations (7, 8, 9, $10,11)$. Longitudinal studies have shown that depression was associated with the incidence and progression of DR, particularly progression to proliferative DR $(12,13)$.

CD163 is a macrophage and monocyte expressed scavenger receptor $(14,15)$. It was initially identified as a pattern recognition receptor in bacterial infections, and it was shown that interaction between bacterial structures and CD163 led to an increase of proinflammatory cytokines (16). The soluble form, sCD163, is shed during inflammation from CD163 by ADAM-17, the same metalloproteinase that sheds TNF- $\alpha$ (17), which is another inflammatory molecule involved in the development of DR (6). Increased concentrations of sCD163 have been demonstrated in the vitreous of the eye in patients with proliferative DR, and the sCD163 levels were particularly high in patients that had developed preretinal fibrovascular membranes (18). Increased levels of plasma sCD163 have been demonstrated in T1D (19), and in metabolic conditions such as obesity, dyslipidemia, hypertension and insulin resistance $(15,20,21,22)$. There are currently no reports on sCD163 in diabetes patients with depressive disorders.

In these patients with $\mathrm{T} 1 \mathrm{D}$, we have previously shown that depression was associated with increased galectin-3 (23), a soluble $\beta$-galactoside-binding lectin, which is expressed in several cells such as activated macrophages and microglia, and is implicated in a variety of biological processes including neuroinflammation and neurodegeneration $(24,25,26)$.

We hypothesize (1) there are immunological links between depression and DR, (2) high levels of plasma sCD163 could reflect the presence of DR and (3) macrophage activation could lead to simultaneous increase in galectin-3 and sCD163 levels. The primary aim was to explore whether high plasma sCD163 levels were associated with depression and with DR. The secondary aim was to explore whether high levels of sCD163 were associated with high galectin-3 levels. While exploring these aims, we controlled for gender, metabolic factors, creatinine, other diabetes complications, life style and medication.

\section{Subjects and methods}

This study has a cross-sectional design and is one of seven baseline analyses $(7,11,23,27,28,29)$ for a randomized controlled trial (ClinicalTrials.gov: NCT01714986) where 'Affect School with Script Analysis' was tried against 'Basic Body Awareness Therapy' for persons with diabetes, inadequate glycemic control and psychological symptoms (30). Patients who attend the clinic every 6 months for regular follow-up visits were consecutively recruited by specialist diabetes physicians or diabetes nurses during a nine-month period, $03 / 25 / 2009$ to $12 / 28 / 2009$, from one hospital diabetes outpatient clinic. The catchment population was 125,000 in Region Kronoberg, Sweden. Inclusion and exclusion criteria, number of participants and missing values are shown in Figure 1. Inclusion criteria were T1D with $\geq 1$-year duration, age $18-59$ years. Exclusion criteria were cancer, hepatic failure, endstage renal disease, psychotic disorder, bipolar disorder, severe personality disorder, severe substance abuse, cognitive deficiency (due to stroke, dementia or mental retardation), or inadequate knowledge of Swedish. There were sCD163 measurements available for 287 patients out of 433 eligible patients (Fig. 1). Thus, 287 patients were included in this study. A questionnaire was used to assess self-reported depression. Blood samples, anthropometrics and blood pressure (BP) were collected. Data were collected from electronic medical records, and from the Swedish National Diabetes Register (S-NDR) (5). All participants provided written informed consent. The study was conducted in accordance with the principles of the Declaration of Helsinki as revised in 2008 and was approved by the Regional Ethical Review Board of Linköping University, Linköping (Registration no. M120-07, T89-08).

\section{Self-reported depression}

Self-reported depression was assessed by the Hospital Anxiety and Depression Scale-Depression subscale (HADS-D) which consists of seven statements, with four response alternatives from 0 to 3 . The cut-off level, $\geq 8$ points, was used (31).

\section{Biochemical analyses}

Blood samples were collected into EDTA plasma tubes (BD, Franklin Lakes, NJ, USA). Plasma sCD163 was analyzed using commercially available human enzymelinked immunosorbent assay (ELISA) DuoSet kits and

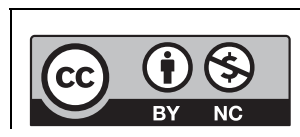

This work is licensed under a Creative Commons Attribution-NonCommercial 4.0 International License. 


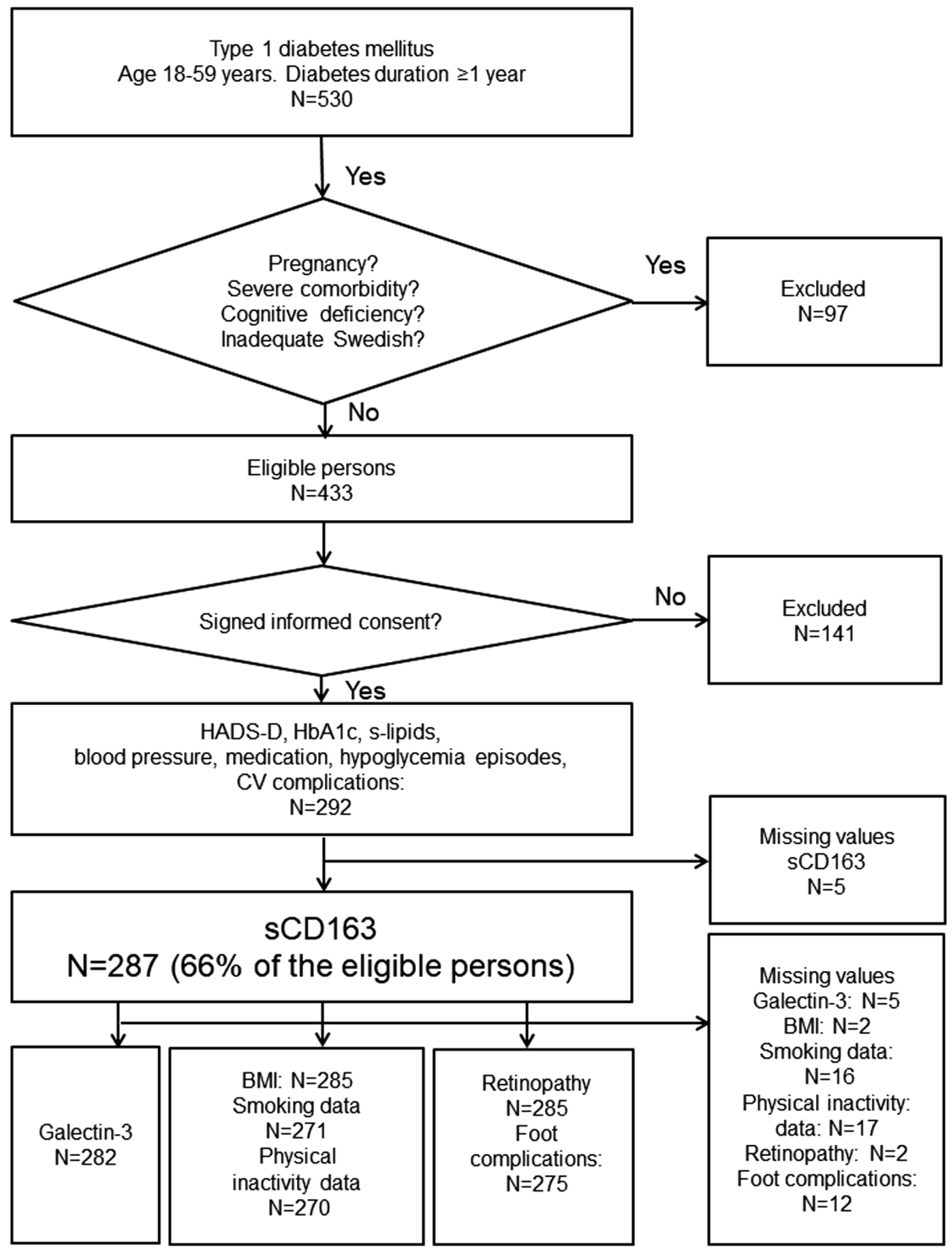

Figure 1

Flow chart describing the 287 included participants, included variables and numbers of missing values.

supplementary ancillary kits (R\&D Systems). Plasma samples from the participants were diluted 1:200 in PBS with $1 \%$ BSA and run in duplicates according to the manufacturer's instructions. Absorbance was measured at $450-580 \mathrm{~nm}$ in a FLOUstar optima plate reader (BMG Labtech $\mathrm{GmbH}$, Ortenberg, Germany). A 7-point standard curve was used to create a 4-parameter logistic regression curve from which concentrations of unknown samples were calculated. The intra-assay coefficient of variation for the sCD163 ELISA analysis was 2.0\%.

The plasma sCD163 (mg/L) were dichotomized at five levels, the 75th; the 80th; the 85th; the 90th; and the 95th percentiles, which were all tried against DR and depression.

The plasma galectin-3 was analyzed using commercial ELISA DuoSet kits and supplementary ancillary kit
(R\&D Systems). Patient samples were diluted 1:2 in PBS supplemented with 1\% BSA and run in duplicates. The ELISA analysis was performed according to the manufacturer's instructions. Absorbance was measured at $450-580 \mathrm{~nm}$ in a FLOUstar optima plate reader (BMG Labtech GmbH, Ortenberg, Germany). A 7-point standard curve was used to create a 4-parameter logistic regression curve from which concentrations of unknown samples were calculated. The intra-assay coefficient of variation for the analysis was $4.3 \%$. Galectin-3 $(\mu \mathrm{g} / \mathrm{L})$ was available for 282 patients and was dichotomized at the 75th; the 80th; the 85th; the 90th and the 95th percentiles, which were tried against the 80th percentile of sCD163.

Venous HbA1c (mmol/mol (\%)) was analyzed with HPLC - variant II, Turbo analyzer (Bio-Rad, Hercules, CA, USA).

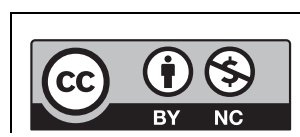

This work is licensed under a Creative Commons Attribution-NonCommercial 4.0 International License. 
Total cholesterol (TC), triglycerides, LDL and HDL $(\mathrm{mmol} / \mathrm{L})$ were collected after an overnight fast, and was measured directly, using the enzymatic color test (Olympus AU).

Creatinine $(\mu \mathrm{mol} / \mathrm{L})$ was assayed by an AU2700 instrument (Beckman Coulter).

\section{Anthropometrics and BP}

Weight, length and BP were measured according to standard procedures by a nurse and BMI $\left(\mathrm{kg} / \mathrm{m}^{2}\right)$ was calculated.

\section{Episodes of hypoglycemia}

A severe episode of hypoglycemia was defined as needing help from another person. Episodes during the last 6 months prior to recruitment were registered.

\section{Smoking and physical inactivity}

Smokers were defined as having smoked any amount of tobacco during the last year.

Physical inactivity was defined as moderate activities, such as $30 \mathrm{~min}$ of walking, less than once a week.

\section{Diabetic retinopathy}

DR was defined as non-proliferative or proliferative retinopathy with microangiopathy changes as viewed by fundus photography through a dilated pupil. Data regarding DR were collected from the S-NDR, where DR disease severity levels were not described in 2009.

\section{Cardiovascular complications}

Cardiovascular complications were defined as ischemic heart disease, cardiac failure, stroke or transient ischemic attack.

\section{Foot complications}

These were defined as neuropathy, angiopathy, earlier or present diabetes foot ulcer, foot infection, foot deformity, arthropathy or amputation of the lower limb.

\section{Medication}

Antidepressants were selective serotonin reuptake inhibitors, serotonin-norepinephrine reuptake inhibitors and/or specific serotonergic antidepressants (N06AB, N06AX16 and N06AX11).

Diabetes-specific treatment was divided into two groups: Insulin only (multiple daily insulin injections or continuous subcutaneous insulin infusion) or insulin combined with oral antidiabetic drugs (OAD) (ATC code A10BA02). The indications for OAD prescription in addition to insulin were obesity and insulin resistance.

Lipid-lowering drugs (LLD) were hydroxymethylglutaryl coenzyme A (HMG CoA) reductase inhibitors (statins) (C10AA). Indications for LLD were TC $>4.5 \mathrm{mmol} / \mathrm{L}(>1.74 \mathrm{mg} / \mathrm{dL})$ and $/$ or $\mathrm{LDL}>2.5 \mathrm{mmol} / \mathrm{L}$ (>97 mg/dL) according to local and Swedish National Guidelines in 2009 (29).

Antihypertensive drugs (AHD) were calcium antagonists (ATC codes C08CA01-02); angiotensinconverting enzyme inhibitors (ATC codes C09AA-BA); angiotensin II antagonists (ATC codes C09CA-DA); diuretics (ATC code C03A) and/or selective betaadrenoreceptor antagonists (ATC code C07AB). Indications for AHD were systolic BP $(\mathrm{SBP})>130 \mathrm{mmHg}$ and/or diastolic BP (DBP) $>80 \mathrm{mmHg}$ according to local and the Swedish National Guidelines in 2009 (29).

\section{Statistical analysis}

Analysis of data distribution using histograms and calculation of skewness was performed and revealed that plasma sCD163 and galectin-3 were not normally distributed. Data were presented as median values (quartile $(\mathrm{q})_{1}, \mathrm{q}_{3}$ ), and analyses were performed with Mann-Whitney $U$ test. Fisher's Exact Test (two-tailed) was used to analyze categorical data. Log-transformation was performed for both sCD163 and galectin-3 when entered in linear regression analyses. Plasma sCD163 and galectin-3 were dichotomized at five cut-off levels corresponding to the 75th, 80th, 85th, 90th and 95th percentiles of each molecule. To establish cut-off levels for defining high levels of sCD163 and galectin-3, two backward elimination multiple logistic regression analyses were performed. The results with the lowest $P$ values were defined as high levels. First, the five cut-off levels for sCD163 were tested against DR. The lowest $P$ value was found for the 80th percentile of sCD163 ( $\geq 0.575 \mathrm{mg} / \mathrm{L})$ which was defined as high sCD163. Second, the five cut-off levels for galectin-3 were tested against sCD163 $\geq 0.575 \mathrm{mg} / \mathrm{L}$. The lowest $P$ value was found for the 95th percentile of galectin-3 $(\geq 4.659 \mu \mathrm{g} / \mathrm{L})$, which was defined as high galectin-3.

Crude odds ratios (CORs) were calculated and variables with $P<0.10$, and gender, age and self-reported https://ec.bioscientifica.com

https://doi.org/10.1530/EC-18-0336
C) 2018 The authors Published by Bioscientifica Ltd
This work is licensed under a Creative Commons Attribution-NonCommercial 4.0 International License. 
depression independent of $P$ values, were entered in multiple logistic regression analyses (Backward: Wald) with high sCD163 and use of antidepressants as dependent variables. The Hosmer and Lemeshow test for goodness of fit and Nagelkerke $R^{2}$ were used to evaluate the multiple logistic regression analyses models. Linear regression was performed with log-transformed galectin-3 and sCD163 data. CIs of 95\% were used. Imputation for one missing value in the HADS-D scale was performed using multinomial regression on the other variables in the scale in one case. $P<0.05$ was considered statistically significant. SPSS version 18 (IBM) was used to perform all statistical analyses.

\section{Results}

Baseline characteristics are presented for all the 287 T1D patients and gender specified in Table 1. The prevalence of foot complications was higher in the 162 men than in the 125 women $(P=0.023)$. Otherwise there were no gender differences.

The test results from HADS-D, the laboratory measurements, the anthropometrics and BP measurements are presented in Table 2 for all and gender specified. S-creatinine, SBP and DBP were higher (all $P<0.001)$, and HDL was lower $(P=0.003)$ in the men.

The results for the sCD163 measurements were positively skewed 2.8 (s.E. 0.1). After log-transformation the skewness was 0.6 (s.e. 0.1). The results for the galectin-3 measurements were positively skewed 11.8 (s.E. 0.1). After log-transformation the skewness was negative -1.9 (s.e. 0.1). Five patients had galectin-3 values $\leq 0.001 \mu \mathrm{g} / \mathrm{L}$.
If these five outliers were excluded the skewness was 0.4 (s.E. 0.1).

Log-transformed sCD163 and log-transformed galectin-3 were associated $(n=282, B$ coefficient 0.046 , $P=0.005$ ) (Fig. 2). Without the five outliers with galectin-3 values $\leq 0.001 \mu \mathrm{g} / \mathrm{L}$, the association between logtransformed sCD163 and log-transformed galectin-3 was higher ( $n=277, B$ coefficient $0.079, P<0.001$ ).

In Table 3 the associations between DR and five cut-off values for sCD163 are presented. High sCD163 ( $\geq 0.575 \mathrm{mg} / \mathrm{L}$ ) (corresponding to the 80th percentile) was associated with DR (AOR 2.4, $P=0.028$ ). The associations between high sCD163 and five cut-off levels for galectin-3 are also presented in Table 3. High galectin-3 ( $\geq 4.659 \mu \mathrm{g} / \mathrm{L}$ ) (corresponding to the 95th percentile) was associated with high sCD163 $(\geq 0.575 \mathrm{mg} / \mathrm{L})$ (AOR 6.1, $P=0.001)$.

The five cut-off values for sCD163, corresponding to the 75 th, 80th, 85 th, 90th and 95th percentiles, were tried against depression, but none of these cut-off levels was associated with depression. The lowest $P$ value was found for the 75th percentile (AOR $1.9(0.8-4.1), P=0.13)$.

Median $\left(\mathrm{q}_{1}, \mathrm{q}_{3}\right)(\mathrm{mg} / \mathrm{L})$ sCD163 were for those using antidepressants $0.56(0.40,0.70) /$ no use of antidepressants $0.42(0.34,0.52), P=0.004$; those with high galectin-3 $0.59(0.42,0.97) /$ low galectin-3 0.42 (0.34, 0.53), $P=0.007$; having DR 0.51 (0.37, $0.64 /$ no DR $0.43(0.34,0.53), P=0.008$; having depression 0.46 $(0.32,0.60) /$ no depression $0.43(0.34,0.53), P=0.66$. Median sCD163 did not differ for the following variables, sorted according to ascending order for the $P$ values ( $P$ values were between 0.063 and 0.64 ): hypoglycemia/no hypoglycemia; OAD/no OAD; AHD/no AHD; smoking/no

Table 1 Baseline characteristics presented for all the 287 T1D patients and comparisons between the 162 men and the 125 women.

\begin{tabular}{l}
\hline$n$ \\
Age (years) \\
Diabetes duration (years) \\
Hypoglycemia (severe episodes) \\
Smoking \\
Physical inactivity \\
Antidepressants \\
Oral anti diabetic drugs \\
Lipid lowering drugs \\
Antihypertensive drugs \\
Cardiovascular complications \\
Diabetic retinopathy \\
Foot complications
\end{tabular}

\begin{tabular}{l}
\hline \multicolumn{1}{c}{ All } \\
\hline 287 \\
$42(31,51 ; 18-59)$ \\
$20(11,30 ; 1-55)$ \\
$12(4)$ \\
$28(10)$ \\
$31(12)$ \\
$22(8)$ \\
$16(6)$ \\
$132(46)$ \\
$96(33)$ \\
$10(4)$ \\
$206(72)$ \\
$47(17)$ \\
\hline
\end{tabular}

\begin{tabular}{c}
\hline Men \\
\hline $162(56)$ \\
$43(32,52)$ \\
$20(11,32)$ \\
$7(4)$ \\
$18(12)$ \\
$18(12)$ \\
$11(7)$ \\
$6(4)$ \\
$78(48)$ \\
$61(38)$ \\
$6(4)$ \\
$118(73)$ \\
$34(22)$ \\
\hline
\end{tabular}

\begin{tabular}{c}
\hline Women \\
\hline $125(44)$ \\
$41(30,50)$ \\
$19(10,29)$ \\
$5(4)$ \\
$10(8)$ \\
$13(11)$ \\
$11(9)$ \\
$10(8)$ \\
$54(43)$ \\
$35(28)$ \\
$4(3)$ \\
$88(71)$ \\
$13(11)$ \\
\hline
\end{tabular}

\begin{tabular}{c}
\hline value $^{\mathrm{a}}$ \\
\hline- \\
$0.11^{\mathrm{b}}$ \\
$0.23^{\mathrm{b}}$ \\
$>0.99$ \\
0.43 \\
$>0.99$ \\
0.66 \\
0.13 \\
0.47 \\
0.10 \\
$>0.99$ \\
0.69 \\
0.023
\end{tabular}

Data are presented as $N(\%)$, median $\left(q_{1}, q_{3} ; \min -\max \right)$, or median $\left(q_{1}, q_{3}\right)$. a Fisher's Exact Test unless otherwise indicated; b Mann-Whitney $U$ test. Missing values: $N={ }^{\mathrm{c}} 16 ;{ }^{\mathrm{d}} 17 ;{ }^{\mathrm{e}} 2 ;{ }^{\mathrm{f}} 12$.

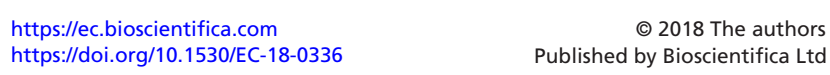


Table 2 Depression, laboratory test results, BMI, and blood pressure in 287 T1D patients and comparisons between men and women.

\begin{tabular}{l}
\hline \\
\hline$n$ \\
Depression (HADS-D $\geq 8)$ \\
sCD163 $(\mathrm{mg} / \mathrm{L})$ \\
High sCD163 $(\geq 0.575 \mathrm{mg} / \mathrm{L})$ \\
Galectin-3 $(\mu \mathrm{g} / \mathrm{L})^{c}$ \\
High Galectin-3 $(\geq 4.659 \mu \mathrm{g} / \mathrm{L})^{c}$ \\
HbA1c \\
$\quad(\mathrm{mmol} / \mathrm{mol})$ \\
$\quad(\%)$ \\
Total cholesterol $(\mathrm{mmol} / \mathrm{L})$ \\
Low density lipoprotein $(\mathrm{mmol} / \mathrm{L})$ \\
Triglycerides $(\mathrm{mmol} / \mathrm{L})$ \\
High density lipoprotein $(\mathrm{mmol} / \mathrm{L})$ \\
S-Creatinine $(\mu \mathrm{mol} / \mathrm{L})$ \\
BMI $\left(\mathrm{kg} / \mathrm{m}^{2}\right)$ \\
Systolic BP $(\mathrm{mmHg})$ \\
Diastolic BP $(\mathrm{mmHg})$
\end{tabular}

\begin{tabular}{l}
\hline \multicolumn{1}{c}{ All } \\
\hline 287 \\
$30(10)$ \\
$0.43(0.34,0.53 ; 0.155-1.901)$ \\
$57(20)$ \\
$0.94(0.57,1.68 ; 0.001-100.0)$ \\
$14(5)$ \\
$63(54,71 ; 25-110)$ \\
$7.9(7.1,8.6 ; 4.4-12.2)$ \\
$4.6(4.1,5.2 ; 2.1-10.9)$ \\
$2.8(2.4,3.3 ; 0.6-8.3)$ \\
$0.9(0.7,1.3 ; 0.1-5.9)$ \\
$1.5(1.3,1.8 ; 0.3-2.7)$ \\
$70(62,78 ; 28-182)$ \\
$25(23,27 ; 18-45)$ \\
$120(110,130 ; 90-160)$ \\
$70(70,75 ; 55-100)$ \\
\hline
\end{tabular}

\begin{tabular}{c}
\hline Men \\
\hline $162(56)$ \\
$16(10)$ \\
$0.44(0.34,0.53)$ \\
$30(18)$ \\
$0.95(0.59,2.04)$ \\
$11(7)$ \\
$62(54,69)$ \\
$7.8(7.1,8.5)$ \\
$4.5(4.1,5.1)$ \\
$2.8(2.4,3.3)$ \\
$0.9(0.7,1.3)$ \\
$1.4(1.2,1.7)$ \\
$74(68,82)$ \\
$25(23,27)$ \\
$125(120,130)$ \\
$70(70,80)$ \\
\hline
\end{tabular}

\begin{tabular}{ccc}
\hline Women & & $\boldsymbol{P}$ value $^{\mathrm{a}}$ \\
\cline { 1 - 1 } $125(44)$ & & - \\
$14(11)$ & & 0.85 \\
$0.42(0.33,0.56)$ & & $0.75^{\mathrm{b}}$ \\
$27(22)$ & & 0.55 \\
$0.93(0.53,1.45)$ & & $0.16^{\mathrm{b}}$ \\
$3(2)$ & & 0.16 \\
& & \\
$64(54,72)$ & & $0.32^{\mathrm{b}}$ \\
$8.0(7.1,8.7)$ & & \\
$4.6(4.1,5.4)$ & & $0.092^{\mathrm{b}}$ \\
$2.9(2.4,3.4)$ & & $0.63^{\mathrm{b}}$ \\
$0.8(0.7,1.3)$ & & $0.46^{\mathrm{b}}$ \\
$1.6(1.5,1.8)$ & & $0.003^{\mathrm{b}}$ \\
$62(54,70)$ & & $<0.001^{\mathrm{b}}$ \\
$25(23,28)$ & & $0.72^{\mathrm{b}}$ \\
$120(110,130)$ & $<0.001^{\mathrm{b}}$ \\
$70(65,75)$ & & $<0.001^{\mathrm{b}}$ \\
\hline
\end{tabular}

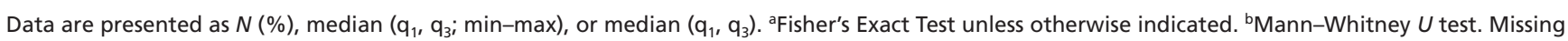
values: $N={ }^{\mathrm{C}} 5 ;{ }^{\mathrm{d}} 2$.

smoking; cardiovascular complications/no cardiovascular complications; foot complications/no foot complications; LLD/no LLD; physical inactivity/no physical inactivity.

In Table 4 patients with high and low sCD163 are compared. Patients with high sCD163 had higher prevalence of antidepressants use $(P=0.004)$, DR $(P=0.031)$ and AHD $(P=0.041)$, and they had higher levels of galectin-3 $(P=0.015)$ and SBP $(P=0.031)$, but lower HDL $(P=0.022)$.

In Table 5 associations with high sCD163 are presented for all and gender specified. In all the 277 T1D patients, high galectin-3 (AOR 9.7), use of antidepressants

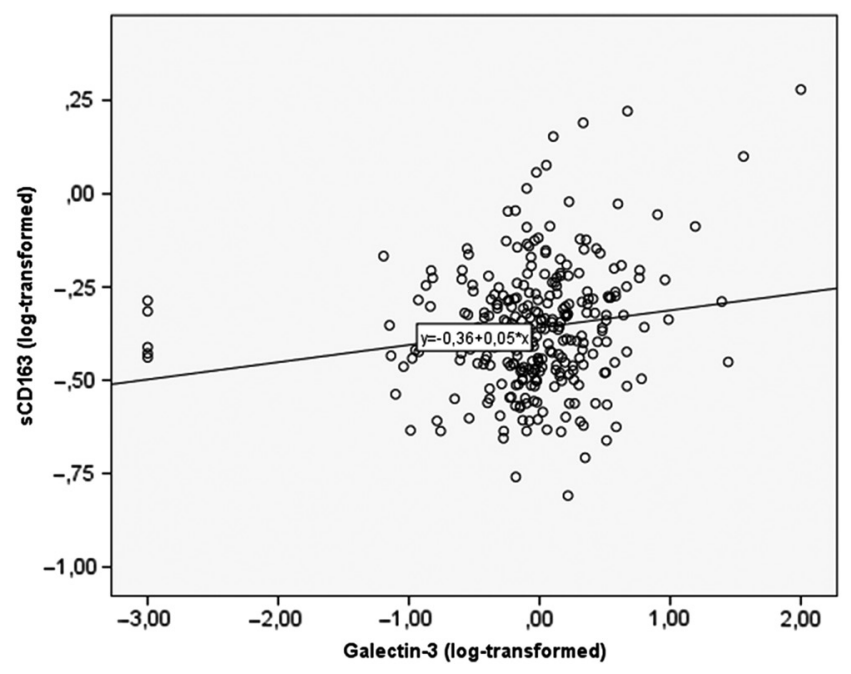

Figure 2

Associations between log-transformed levels of galectin-3 and sCD163.

https://ec.bioscientifica.com

https://doi.org/10.1530/EC-18-0336

(C) 2018 The authors Published by Bioscientifica Ltd
(AOR 3.8), SBP (per mmHg) (AOR 1.03) and DR (AOR 2.4) were associated with high sCD163. In the 160 men, high galectin-3 (AOR 13.1), SBP (per mmHg) (AOR 1.05) and DR (AOR 7.1) were associated with high sCD163. In the 123 women, the use of antidepressants (AOR 5.5) was associated with high sCD163.

Of the 22 patients that used antidepressants, 10 (46\%) had self-reported depression and $12(54 \%)$ patients did not and of the 265 patients that did not use antidepressants, 20 (8\%) had self-reported depression and $245(92 \%)$ did not.

Twenty (10\%) patients out of 209 with DR, and three (4\%) patients out of 81 without DR used antidepressants $(P=0.14)$.

Depression $\quad($ AOR $9.2 \quad(3.2-26.2), \quad P<0.001)$, cardiovascular complications (AOR 10.2 (2.3-44.2), $P=0.002$ ) and high sCD163 (AOR $3.6(1.3-10.1), P=0.014$ ) were associated with the use of antidepressants. No other factors included in the study were associated with the use of antidepressants ( $P$-values $\geq 0.13$ ). Nagelkerke R Square was 0.290 and the Hosmer and Lemeshow test was 0.869 .

\section{Discussion}

The main findings in this study of depression, diabetes retinopathy, sCD163 and galectin-3 in patients with T1D, were that DR, high levels of galectin-3 $(\geq 4.659 \mu \mathrm{g} / \mathrm{L})$, use of antidepressants and increasing levels of SBP,

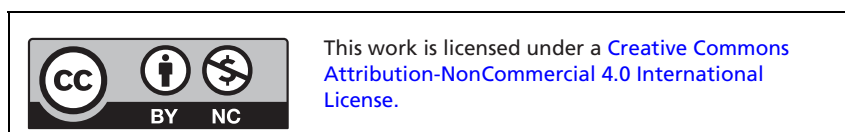


Table 3 Analyses performed to establish suitable cut-off levels for high sCD163 and high galectin-3.

sCD163 $\geq 0.534^{c}(\geq 75 \text { th })^{d}$
$s C D 163 \geq 0.575^{c}(\geq 80 \text { th })^{d}$
$s C D 163 \geq 0.613^{c}(\geq 85 t h)^{d}$
$s C D 163 \geq 0.697^{c}(\geq 90 \text { th })^{d}$
$s C D 163 \geq 0.819^{c}(\geq 95 \text { th })^{d}$
Galectin-3 $\geq 1.681^{e}(\geq 75 t h)^{d}$
Galectin-3 $\geq 2.102^{e}(\geq 80 \text { th })^{d}$
Galectin-3 $\geq 2.562^{e}(\geq 85 t h)^{d}$
Galectin-3 $\geq 3.272^{e}(\geq 90 t h)^{d}$
Galectin-3 $\geq 4.659^{e}(\geq 95 \text { th })^{d}$

\begin{tabular}{|c|c|c|c|c|}
\hline \multicolumn{5}{|c|}{ Diabetic retinopathy } \\
\hline Yes & No & $P^{a}$ & AOR (CI) & $P$ value $^{\mathrm{b}}$ \\
\hline $57(79)$ & $15(21)$ & 0.17 & $0.6(0.2-1.9)$ & 0.42 \\
\hline $48(84)$ & $9(16)$ & 0.031 & $2.4(1.1-5.1)$ & 0.028 \\
\hline $36(84)$ & $7(16)$ & 0.095 & $1.0(0.2-6.5)$ & 0.96 \\
\hline $23(82)$ & $5(20)$ & 0.27 & $0.9(0.1-7.6)$ & 0.94 \\
\hline $11(79)$ & $3(21)$ & 0.76 & $0.6(0.1-2.8)$ & 0.51 \\
\hline - & - & - & - & - \\
\hline - & - & - & - & - \\
\hline - & - & - & - & - \\
\hline - & - & - & - & - \\
\hline - & - & - & - & - \\
\hline
\end{tabular}

\begin{tabular}{|c|c|c|c|c|}
\hline \multicolumn{5}{|c|}{ High sCD163 ( $\geq 0.575$ mg/L) } \\
\hline Yes & No & $P^{a}$ & AOR (CI) & $P$ value $^{\mathrm{b}}$ \\
\hline- & - & - & - & - \\
\hline- & - & - & - & - \\
\hline- & - & - & - & - \\
\hline - & - & - & - & - \\
\hline - & - & - & - & - \\
\hline $19(26)$ & $53(74)$ & 0.12 & $1.1(0.3-4.0)$ & 0.91 \\
\hline $16(29)$ & $40(71)$ & 0.09 & $1.3(0.3-4.8)$ & 0.72 \\
\hline $13(31)$ & $29(69)$ & 0.06 & $0.8(0.2-3.5)$ & 0.73 \\
\hline $11(39)$ & $17(61)$ & 0.011 & $1.3(0.3-4.7)$ & 0.72 \\
\hline $8(57)$ & $6(43)$ & 0.002 & $6.1(2.0-18.4)$ & 0.001 \\
\hline
\end{tabular}

${ }^{a}$ Fisher's Exact Test; ${ }^{b}$ multiple logistic regression analysis (Backward: Wald); ${ }^{c} \mathrm{mg} / \mathrm{L} ;{ }^{d}$ percentile; ${ }^{\mathrm{e}} \mu \mathrm{g} / \mathrm{L}$.

were independently associated with high sCD163 $(\geq 0.575 \mathrm{mg} / \mathrm{L})$. Depression was not associated with high sCD163. Gender sub-analyses showed that DR, high galectin-3 and increasing levels of SBP were independently associated with high sCD163 in the men. In the women, the use of antidepressants was independently associated with high sCD163.
Strengths of the study were that we can demonstrate new significant findings. The association between plasma sCD163 and plasma galectin-3 was strong and has not to our knowledge been demonstrated previously. This is of interest as both molecules are released from macrophages, and both indicate increased inflammatory responses $(15,21,24,25,26,32)$. Another new finding is the

Table 4 Comparisons between the 57 patients with high levels of sCD163 and the 230 patients with low levels.

\begin{tabular}{|c|c|c|c|}
\hline & \multicolumn{3}{|c|}{ sCD163 (mg/L) } \\
\hline & High levels $(\geq 0.575)$ & Low levels $(<0.575)$ & $P$ value ${ }^{\mathrm{a}}$ \\
\hline$n$ & 57 & 230 & \\
\hline \multicolumn{4}{|l|}{ Gender } \\
\hline Men & $30(53)$ & $132(57)$ & 0.55 \\
\hline Women & $27(47)$ & $98(43)$ & \\
\hline Age (years) & $41(34,54)$ & $42(31,51)$ & $0.34^{\mathrm{b}}$ \\
\hline Diabetes duration (years) & $22(18,29)$ & $19(10,30)$ & $0.13^{b}$ \\
\hline Depression (HADS-D $\geq 8$ ) & $8(14)$ & $22(10)$ & 0.34 \\
\hline Galectin-3 $(\mu \mathrm{g} / \mathrm{L})^{\mathrm{c}}$ & $1.2(0.8,2.2)$ & $0.9(0.5,1.6)$ & $0.015^{b}$ \\
\hline \multicolumn{4}{|l|}{$\mathrm{HbA} 1 \mathrm{c}$} \\
\hline$(\mathrm{mmol} / \mathrm{mol})$ & $65(55,74)$ & $63(54,71)$ & $0.17^{b}$ \\
\hline$(\%)$ & $8.1(7.2,8.9)$ & $7.9(7.1,8.6)$ & \\
\hline Total cholesterol (mmol/L) & $4.6(4.0,5.4)$ & $4.6(4.1,5.1)$ & $0.50^{\mathrm{b}}$ \\
\hline Low density lipoprotein (mmol/L) & $2.9(2.4,3.6)$ & $2.8(2.4,3.2)$ & $0.18^{b}$ \\
\hline Triglycerides (mmol/L) & $1.0(0.7,1.5)$ & $0.9(0.7,1.2)$ & $0.18^{b}$ \\
\hline High density lipoprotein (mmol/L) & $1.4(1.3,1.6)$ & $1.6(1.3,1.8)$ & $0.022^{b}$ \\
\hline S-Creatinine $(\mu \mathrm{mol} / \mathrm{L})$ & $69(58,79)$ & $70(62,78)$ & $0.50^{\mathrm{b}}$ \\
\hline$B M I^{d}\left(k g / m^{2}\right)$ & $26(23,28)$ & $25(23,27)$ & $0.14^{b}$ \\
\hline Systolic BP (mmHg) & $130(112,135)$ & $120(110,130)$ & $0.031^{b}$ \\
\hline Diastolic BP (mmHg) & $70(70,78)$ & $70(65,75)$ & $0.21^{\mathrm{b}}$ \\
\hline Hypoglycemia & $1(2)$ & $11(5)$ & 0.47 \\
\hline Smoking & $4(8)$ & $24(11)$ & 0.62 \\
\hline Physical inactivity ${ }^{f}$ & $5(9)$ & $26(12)$ & 0.81 \\
\hline Antidepressants & $10(18)$ & $12(5)$ & 0.004 \\
\hline Oral anti diabetic drugs & $5(9)$ & $11(5)$ & 0.33 \\
\hline Lipid lowering drugs & $24(42)$ & $108(47)$ & 0.56 \\
\hline Antihypertensive drugs & $26(46)$ & $70(30)$ & 0.041 \\
\hline Cardiovascular complications & $3(5)$ & $7(3)$ & 0.42 \\
\hline Diabetic retinopathyg & $48(84)$ & $158(69)$ & 0.031 \\
\hline Foot complications ${ }^{\mathrm{h}}$ & $11(20)$ & $36(16)$ & 0.54 \\
\hline
\end{tabular}

Data are presented as median $\left(q_{1}, q_{3}\right)$ or $N(\%)$. ${ }^{a}$ Fisher's Exact Test unless otherwise indicated. b Mann-Whitney $U$ test. Missing values: $N={ }^{c 5}$; ${ }^{\mathrm{d}}$; ${ }^{\mathrm{e}} 16$; ${ }^{\mathrm{f}} 17 ;{ }^{9} 2 ;{ }^{\mathrm{h}} 12$.

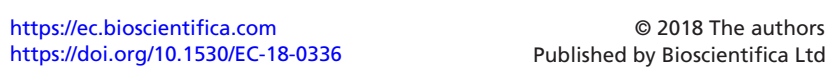


Table 5 Associations with high sCD163 for all patients and gender specified.

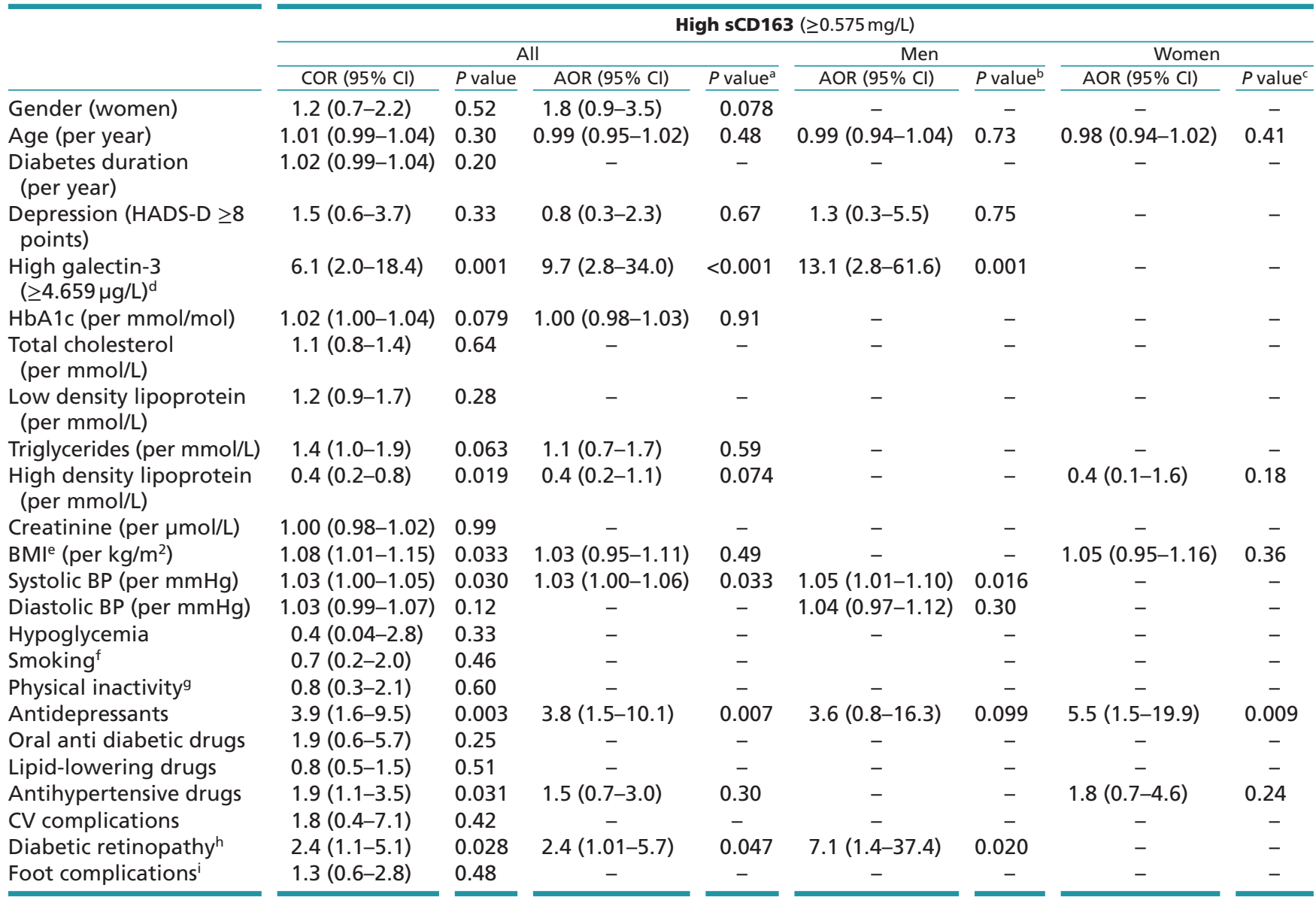

a,b,c Multiple logistic regression analysis (Backward Wald). $N={ }^{\mathrm{a}} 277 / \mathrm{b} 160 / 123$. Nagelkerke R Square ${ }^{\mathrm{a}} 0.185 / \mathrm{b} 0.279 /{ }^{\mathrm{c}} 0.081$. Hosmer and Lemeshow: ${ }^{\mathrm{a}} 0.791 /{ }^{\mathrm{b}} 0.355 /{ }^{\mathrm{C}} 0.301$. Missing values: $N={ }^{\mathrm{d}} 5 ;{ }^{\mathrm{e}} 2 ;{ }^{\mathrm{f}} 16 ;{ }^{9} 17 ;{ }^{\mathrm{h}} 2 ;{ }^{\mathrm{i}} 12$.

association between circulating sCD163 and DR, which has not been explored previously. This is important as high levels of sCD163 have in previous research been demonstrated in the vitreous of the eye, particularly in patients with severe proliferative types of DR (18). A third new finding is the association between the use of antidepressants and sCD163.

Another strength is that precise ELISA techniques were used. The commercial ELISA assay showed a low intra-assay coefficient of variation for both sCD163 and galectin-3.

Further, the population of patients with T1D was well defined, since persons with severe somatic and psychiatric comorbidities and severe substance abuse were excluded. We have systematically controlled for potential confounders, and we have explored gender differences.

One difficulty we had to address was that there is no established consensus regarding harmful plasma sCD163 levels. Therefore, we explored the associations between DR and five cut-off levels (from the 75th to the 95th percentile) of sCD163 to find the optimal cut-off level for sCD163, which could have a clinical implication. We also systematically explored the associations between high sCD163 and five cut-off levels for galectin-3. Here we found that high galectin-3, using a cut-off level corresponding to the 95th percentile, was associated with high sCD163. Previously we have found that a lower cut-off level, corresponding to the 85th percentile of galectin-3, was associated with depression (23). Finally, we explored the associations between depression and the five cut-off levels for sCD163, without finding any association at any level.

The main limitation of our study was that the DR was not categorized according to severity. The reason is that we collected the information regarding DR from the S-NDR, where disease severity levels were not reported in 2009.

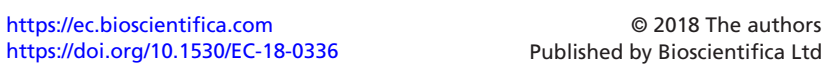


We still consider that the fact that high levels of plasma sCD163 did reflect the presence of DR is worth presenting, even without classification of DR severity. Another weakness was the limited number of patients within certain sub groups. There were only 22 (8\%) patients using antidepressants which prevents analyses of associations between different types of antidepressants and sCD163. The association between the use of antidepressants and high sCD163 did not reach significance for the 11 men, but the $P$ value was below 0.10 , which indicates that this is a possible type 2 error. Depression was not confirmed by a structured interview, but it was recently shown that HADS-D is a useful instrument for detecting symptoms of depression, both at an individual and a collective level, and has been demonstrated to have a good reliability and discriminant validity (31).

We found that high levels of sCD163 was associated with increasing SBP, but not with increasing DBP, which differs from previous research (22). The patients with high levels of sCD163 had a higher prevalence of AHD which might have lowered the DBP more effectively than the SBP. We found no association between BMI and high sCD163, contrarily to in previous research where associations between sCD163 and obesity were described (21). Neither did we find any association between HbA1c and high sCD163, which could have been possible due to the previously demonstrated impact of sCD163 on insulin resistance (20).

There are several subjects for further research. As this is a cross-sectional study, we have no information regarding sCD163 levels at the initiation of antidepressant medication. To be able to draw any conclusions about whether one or several types of antidepressants are causing high sCD163 or not, further research will be necessary. The associations between different types of antidepressants, depression and sCD163 must be further explored in prospective studies. It was unexpected to find a positive association between the use of antidepressants and sCD163, as in previous research it has been demonstrated that antidepressant medication attenuated inflammatory activity $(10,33)$.

In further research we wish to explore whether increasing sCD163 levels are associated with increased severity of DR. We plan a 10-year follow-up of this study, and we will then use information from the medical records to categorize DR according to severity levels both at baseline and at follow-up. It would also be of interest to measure the levels of sCD163 in the vitreous of the eye and in the plasma simultaneously. It is important to find biomarkers that can contribute to the understanding of the pathology of DR, that can be used for early detection, to check the pharmacological response during therapeutic intervention, or that can be used as treatment targets $(2,6,18)$. In previous research it has been shown that glucagon-like peptide 1 (GLP-1) analog therapy directly modulates innate immune-mediated inflammation in individuals with type 2 diabetes mellitus (34). If sCD163 is causative in the development of DR, one potential treatment for reducing sCD163 could be a GLP-1 analog, which would be another subject for further research.

Our main hypothesis was that there are immunological links between depression and DR. We found no evidence that SCD163 was one of these immunological links. Instead we found that the use of antidepressants was associated with high levels of sCD163. Our second hypothesis that high levels of plasma sCD163 could reflect the presence of DR was confirmed. This is of great importance as plasma sCD163, but not vitreous sCD163, might be used to check the pharmacological response during therapeutic interventions. Our third hypothesis was that macrophage activation could lead to simultaneous increase in galectin-3 and sCD163 levels, and we did find a strong association between high galectin-3 and high sCD163. We did not find any association between the use of antidepressants and DR. To our knowledge, this is the first study to explore and to show that circulating SCD163 was associated with circulating galectin-3, the use of antidepressants, DR and SBP in patients with T1D.

\section{Declaration of interest}

The authors declare that there is no conflict of interest that could be perceived as prejudicing the impartiality of the research reported.

\section{Funding}

This research was supported by the Research and Development Fund of Health Care, Region Kronoberg, Växjö, Sweden, and by the Research Council of South Eastern Sweden (FORSS), Linköping, Sweden. The funding sources were not involved in the collection, analysis and interpretation of data, in the writing of the report, or in the decision to submit the article for publication.

\section{Author contribution statement}

E O M, J D, M T and M H participated as investigators and reviewed, edited and approved of the final version of the manuscript. E O M initiated the study of depression in diabetes, performed the statistical analysis, and is the guarantor of this work and, as such, had full access to all the data in the study and takes responsibility for the integrity of the data and the accuracy of the data analysis. M H suggested that SCD1634 would be of interest to study. $\mathrm{EO} \mathrm{M}$ and $\mathrm{M} \mathrm{H}$ wrote the manuscript. J D performed and validated the SCD163 and galectin-3 analyses. E O M and M T organized the recruitment of patients and collection of data. 


\section{Acknowledgements}

The authors are grateful to Anna Lindgren, PhD at the Department of Mathematical Statistics, Lund University, Lund, Sweden, for her statistical skills.

\section{References}

1 Wang W \& Lo A. Diabetic retinopathy: pathoph and treatments. International Journal of Molecular Sciences 201819 E1816. (https://doi. org/10.3390/ijms19061816)

2 McAuley AK, Sanfilippo PG, Hewitt AW, Liang H, Lamoureux E, Wang JJ \& Connell PP. Vitreous biomarkers in diabetic retinopathy: a systematic review and meta-analysis. Journal of Diabetes and its Complications 201428 419-425. (https://doi.org/10.1016/j. jdiacomp.2013.09.010)

3 Cunha-Vaz J, Ribeiro L \& Lobo C. Phenotypes and biomarkers of diabetic retinopathy. Progress in Retinal and Eye Research 201441 90-111. (https://doi.org/10.1016/j.preteyeres.2014.03.003)

4 Capitão M \& Soares R. Angiogenesis and inflammation crosstalk in diabetic retinopathy. Journal of Cellular Biochemistry 2016117 2443-2453. (https://doi.org/10.1002/jcb.25575)

5 Jendle J, Ericsson Å, Hunt B, Valentine WJ \& Pollock RF. Achieving good glycemic control early after onset of diabetes: a costeffectiveness analysis in patients with type 1 diabetes in Sweden. Diabetes Therapy 20189 87-99. (https://doi.org/10.1007/s13300-0170344-6)

6 Khan AA, Rahmani AH \& Aldebasi YH. Diabetic retinopathy: recent updates on different biomarkers and some therapeutic agents. Current Diabetes Reviews 201713 1-11. (https://doi.org/10.2174/1573399813 666170915133253)

7 Melin EO, Thunander M, Svensson R, Landin-Olsson M \& Thulesius HO. Depression, obesity and smoking were independently associated with inadequate glycemic control in patients with type 1 diabetes. European Journal of Endocrinology 2013168 861-869. (https://doi.org/10.1530/eje-13-0137)

8 Penninx BW, Milaneschi Y, Lamers F \& Vogelzangs N. Understanding the somatic consequences of depression: biological mechanisms and the role of depression symptom profile. BMC Medicine 201311129. (https://doi.org/10.1186/1741-7015-11-129)

9 Korczak DJ, Pereira S, Koulajian K, Matejcek A \& Giacca A. Type 1 diabetes mellitus and major depressive disorder: evidence for a biological link. Diabetologia 201154 2483-2493. (https://doi. org/10.1007/s00125-011-2240-3)

10 Raison CL, Capuron L \& Miller AH. Cytokines sing the blues: inflammation and the pathogenesis of depression. Trends in Immunology 200627 24-31. (https://doi.org/10.1016/j. it.2005.11.006)

11 Melin EO, Thunander M, Landin-Olsson M, Hillman M \& Thulesius HO. Depression, smoking, physical inactivity and season independently associated with midnight salivary cortisol in type 1 diabetes. BMC Endocrine Disorders 201414 75. (https://doi. org/10.1186/1472-6823-14-75)

12 Roy MS, Roy A \& Affouf M. Depression is a risk factor for poor glycemic control and retinopathy in African-Americans with type 1 diabetes. Psychosomatic Medicine 200769 537-542. (https://doi. org/10.1097/PSY.0b013e3180df84e2)

13 Sieu N, Katon W, Lin EH, Russo J, Ludman E \& Ciechanowski P. Depression and incident diabetic retinopathy: a prospective cohort study. General Hospital Psychiatry 201133 429-435. (https://doi. org/10.1016/j.genhosppsych.2011.05.021)

14 Kristiansen M, Graversen JH, Jacobsen C, Sonne O, Hoffman HJ, Law SA \& Moestrup SK. Identification of the haemoglobin scavenger receptor. Nature 2001409 198-201. (https://doi. org/10.1038/35051594)
15 Møller HJ. Soluble CD163. Scandinavian Journal of Clinical and Laboratory Investigation 201272 1-13. (https://doi.org/10.3109/00365 513.2011.626868)

16 Fabriek BO, van Bruggen R, Deng DM, Ligtenberg AJ, Nazmi K, Schornagel K, Vloet RP, Dijkstra CD \& van den Berg TK. The macrophage scavenger receptor CD163 functions as an innate immune sensor for bacteria. Blood 2009113 887-892. (https://doi. org/10.1182/blood-2008-07-167064)

17 Etzerodt A, Maniecki MB, Møller K, Møller HJ \& Moestrup SK. Tumor necrosis factor $\alpha$-converting enzyme (TACE/ADAM17) mediates ectodomain shedding of the scavenger receptor CD163. Journal of Leukocyte Biology 201088 1201-1205. (https://doi. org/10.1189/jlb.0410235)

18 Kobayashi Y, Yoshida S, Nakama T, Zhou Y, Ishikawa K, Arita R, Nakao S, Miyazaki M, Sassa Y \& Oshima Y. Overexpression of CD163 in vitreous and fibrovascular membranes of patients with proliferative diabetic retinopathy: possible involvement of periostin. British Journal of Ophthalmology 201599 451-456. (https://doi. org/10.1136/bjophthalmol-2014-30521)

19 Llaurado G, Gonzalez-Clemente JM, Maymo-Masip E, Subías D, Vendrell J \& Chacon MR. Serum levels of TWEAK and scavenger receptor CD163 in type 1 diabetes mellitus: relationship with cardiovascular risk factors. a case-control study. PLOS ONE 20127 e43919. (https://doi.org/10.1371/journal.pone.0043919)

20 Parkner T, Sørensen L, Nielsen A, Fischer C, Bibby BM, Nielsen S, Pedersen B \& Møller H. Soluble CD163: a biomarker linking macrophages and insulin resistance. Diabetologia 201255 1856-1862. (https://doi.org/10.1007/s00125-012-2533-1)

21 Fjeldborg K, Christiansen T, Bennetzen M, J Møller H, Pedersen SB $\&$ Richelsen $\mathrm{B}$. The macrophage-specific serum marker, soluble CD163, is increased in obesity and reduced after dietary-induced weight loss. Obesity 201321 2437-2443. (https://doi.org/10.1002/ oby.20376)

22 Al-Daghri NM, Al-Attas OS, Bindahman LS, Alokail MS, Alkharfy KM, Draz HM, Yakout S, McTernan PG, Sabico S \& Chrousos GP. Soluble CD163 is associated with body mass index and blood pressure in hypertensive obese Saudi patients. European Journal of Clinical Investigation 201242 1221-1226. (https://doi.org/10.1111/j.13652362.2012.02714.x)

23 Melin EO, Dereke J, Thunander M \& Hillman M. Depression in type 1 diabetes was associated with high levels of circulating galectin-3. Endocrine Connections 20187 819-828. (https://doi.org/10.1530/ EC-18-0108)

24 Burguillos MA, Svensson M, Schulte T, Boza-Serrano A, GarciaQuintanilla A, Kavanagh E, Santiago M, Viceconte N, OlivaMartin MJ \& Osman AM. Microglia-secreted galectin-3 acts as a toll-like receptor 4 ligand and contributes to microglial activation. Cell Reports 201510 1626-1638. (https://doi.org/10.1016/j. celrep.2015.02012)

25 Shin T. The pleiotropic effects of galectin-3 in neuroinflammation: a review. Acta Histochemica 2013115 407-411. (https://doi. org/10.1016/j.acthis.2012.11.010)

26 Yip PK, Carrillo-Jimenez A, King P, Vilalta A, Nomura K, Chau CC, Egerton AMS, Liu ZH, Shetty AJ, Tremoleda JL, et al. Galectin-3 released in response to traumatic brain injury acts as an alarmin orchestrating brain immune response and promoting neurodegeneration. Scientific Reports 20177 41689. (https://doi. org/10.1038/srep41689)

27 Melin EO, Thunander M, Landin-Olsson M, Hillman M \& Thulesius HO. Depression differed by midnight cortisol secretion, alexithymia and anxiety between diabetes types: a cross sectional comparison. BMC Psychiatry 201717 335. (https://doi.org/10.1186/ s12888-017-1495-8)

28 Melin EO, Svensson R, Thunander M, Hillman M, Thulesius HO \& Landin-Olsson M. Gender, alexithymia and physical inactivity associated with abdominal obesity in type 1 diabetes mellitus: a cross

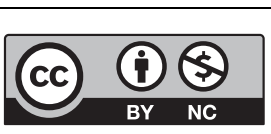

This work is licensed under a Creative Commons Attribution-NonCommercial 4.0 International License. 
sectional study at a secondary care hospital diabetes clinic. $B M C$ Obesity 20174 21. (https://doi.org/10.1186/s40608-017-0157-1)

29 Melin EO, Thulesius HO, Hillman M, Landin-Olsson M \&

Thunander M. Abdominal obesity in type 1 diabetes associated with gender, cardiovascular risk factors and complications, and difficulties achieving treatment targets: a cross sectional study at a secondary care diabetes clinic. BMC Obesity 20185 15. (https://doi.org/10.1186/ s40608-018-0193-5)

30 Melin EO, Svensson R, Gustavsson SÅ, Winberg A, Denward-Olah E, Landin-Olsson M \& Thulesius HO. Affect school and script analysis versus basic body awareness therapy in the treatment of psychological symptoms in patients with diabetes and high HbA1c concentrations: two study protocols for two randomized controlled trials. Trials 2016 17 221. (https://doi.org/10.1186/s13063-016-1347-8)

31 Bocéréan C \& Dupret E. A validation study of the Hospital Anxiety and Depression Scale (HADS) in a large sample of French employees.
BMC Psychiatry 201414 1-11. (https://doi.org/10.1186/s12888-0140354-0)

32 Ingels C, Møller HJ, Hansen TK, Wouters PJ, Vanhorebeek I \& Van den Berghe G. Circulating levels of the shed scavenger receptor sCD163 and association with outcome of critically ill patients. Journal of Clinical Immunology 201333 619-629. (https://doi. org/10.1007/s10875-012-9830-9)

33 Kenis G \& Maes M. Effects of antidepressants on the production of cytokines. International Journal of Neuropsychopharmacology 20025 401-412. (https://doi.org/10.1017/S1461145702003164)

34 Hogan AE, Gaoatswe G, Lynch L, Corrigan MA, Woods C, O'Connell J \& O'Shea D. Glucagon-like peptide 1 analogue therapy directly modulates innate immune-mediated inflammation in individuals with type 2 diabetes mellitus. Diabetologia 201457 781-784. (https://doi.org/10.1007/s00125013-3145-0)

Received in final form 10 October 2018

Accepted 24 October 2018

Accepted Preprint published online 26 October 2018 https://ec.bioscientifica.com

https://doi.org/10.1530/EC-18-0336
() 2018 The authors Published by Bioscientifica Ltd

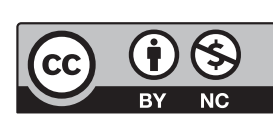

This work is licensed under a Creative Commons Attribution-NonCommercial 4.0 International License. 